\title{
Penetration Depth of Sodium Hypochlorite in Dentinal Tubules after Conventional Irrigation, Passive Ultrasonic Agitation and Nd:YAG Laser Activated Irrigation
}

\author{
Abdollah Ghorbanzadeh ${ }^{1}$, Mohsen Aminsobhani ${ }^{2}$, Khosro Sohrabi ${ }^{3}$, Nasim Chiniforush ${ }^{3}$, Sarvenaz Ghafari ${ }^{4}$, \\ Ahmad Reza Shamshiri ${ }^{5}$, Niusha Noroozi ${ }^{6}$ \\ 'Endodontic Department, School of Dentistry, Tehran University of Medical Sciences, Tehran, Iran \\ ${ }^{2}$ Endodontic Department, School of Dentistry, Tehran University of Medical Sciences and AJA University of Medical Sciences, \\ Tehran, Iran \\ 3Laser Research Center of Dentistry, School of Dentistry, Tehran University of Medical Sciences, Tehran, Iran \\ ${ }^{4}$ Endodontic Department, School of Dentistry, Kermanshah University of Medical Sciences, Kermanshah, Iran \\ ${ }^{5}$ Epidemiologist, Tehran University of Medical Sciences, Tehran, Iran \\ ${ }^{6}$ Private Practice
}

\section{*Correspondence to}

Mohsen Aminsobhani, DDS MSc; Endodontic Department, School of Dentistry, Tehran University of Medical Sciences and AJA University of Medical Sciences, Tehran, Iran; Tel: +9821-88950567;

Fax: +98-21-66431744;

Email:

maminsobhani@yahoo.com

Published online 27 March 2016

\begin{abstract}
Introduction: The penetration depth of irrigating solutions in dentinal tubules is limited; consequently, bacteria can remain inside dentinal tubules after the cleaning and shaping of the root canal system. Therefore, new irrigation systems are required to increase the penetration depth of irrigating solutions in dentinal tubules.

Methods: A comparative study regarding the penetration depth of sodium hypochlorite $(\mathrm{NaOCl})$ solution in dentinal tubules using four methods, (1) conventional irrigation $(\mathrm{Cl})$, (2) smear layer removal plus conventional irrigation (gold standard), (3) passive ultrasonic agitation (PUA) and (4) Nd:YAG laser activated irrigation (LAI), took place on 144 extracted mandibular teeth with a single root canal. After decoronation with a diamond disc and working length determination, the apical foramen was sealed with wax. The canals were prepared up to \#35 Mtwo rotary file and $5.25 \% \mathrm{NaOCl}$ was used for irrigation during preparation. To study the penetration depth of $\mathrm{NaOCl}$, smear layer was eliminated in all samples. Dentinal tubules were stained with crystal violet and after longitudinal sectioning of teeth, the two halves were reassembled and root canal preparation was performed up to \#40 Mtwo rotary file. Then the samples were distributed into four experimental groups. Depth of the bleached zone was evaluated by stereomicroscope (20X). Data were analyzed by Kruskal-Wallis test.

Results: The highest and lowest average for $\mathrm{NaOCl}$ penetration depth in all three coronal, middle and apical sections belonged to $\mathrm{Cl}+$ smear layer removal and $\mathrm{Cl}$. A statistically significant difference was seen when comparing the penetration depth of $\mathrm{Cl}+$ smear layer removal group to $\mathrm{Cl}$ and $\mathrm{PUA}$ groups in coronal and middle third, in which the average $\mathrm{NaOCl}$ penetration depth of the gold standard group was higher $(P<0.05)$. A statistically significant difference was seen between $\mathrm{Cl}+$ smear layer removal group and the other three groups including $\mathrm{Cl}, \mathrm{PUA}$ and $\mathrm{LAI}$ in apical third, in which the average $\mathrm{NaOCl}$ penetration depth in the gold standard group was higher $(P<0.001)$.

Conclusion: The standard protocol for smear layer removal led to more effective smear layer elimination and deeper penetration depth of irrigation solutions. PUA and LAI groups exhibited less smear layer elimination and penetration depth of irrigation solutions. Therefore, $\mathrm{Cl}+$ smear layer removal should still be considered as the gold standard.

Keywords: Agitation; Irrigation; Lasers; Nd:YAG; Ultrasonic.
\end{abstract}

\section{Introduction}

Complete cleaning and shaping of root canal system, is an important part in endodontic treatments, which is performed by mechanical and chemical methods. Elimination of the pulp tissue, mineral and organic debris, micro-organisms and their by-products with the use of inter

Please cite this article as follows: Ghorbanzadeh A, Aminsobhani M, Sohrabi K, et al. Penetration depth of sodium hypochlorite in dentinal tubules after conventional irrigation, passive ultrasonic agitation and Nd:YAG laser activated irrigation. J Lasers Med Sci. 2016;7(2):105111. doi:10.15171/jlms.2016.18. 
canal irrigation instruments is one of the main aims of this treatment phase. ${ }^{1}$ While treating infected root canals, special attention should be guided towards eliminating bacteria and their byproducts from the root canal system. The practicality of irrigating solutions depends on different factors such as the irrigation mechanism, its contact potential with substances, materials and root canal structures, their penetration depth in the main canal and lateral spaces. ${ }^{2}$

When evaluating the practicality of debris elimination in 3 irrigating solutions, Baker et al proposed that the flushing effect was more important than the power of tissue resolution. ${ }^{3}$ Therefore, sodium hypochlorite's efficiency might be affected by the method of use.

For instance, it has been shown that when sodium hypochlorite is activated with ultrasonic, its efficiency increases. ${ }^{4}$ Better penetration of chemical substances, irrigation solutions and intra canal medications into dentinal tubules caused by smear layer removal allows a better disinfection and seal in the root canal system. ${ }^{5}$

Root canal irrigation systems can be divided into two main groups, including hand agitation techniques and agitation techniques with devices. Hand agitation consists of irrigation with positive forces, commonly by the use of syringes and side-vented needles.

Agitation techniques with devices include sonic, ultra sonic, also newer systems like Endo VAC (apical negative pressure $)^{6}$ and plastic rotary files. ${ }^{7,8}$

Paragliola et al concluded that ultrasonic agitation can increase the effect of the final irrigant on root canal walls in the apical third. ${ }^{9}$

The penetration depth of irrigation solutions and therefore their disinfecting effect on dentinal tubules are limited. These limitations lead to incomplete bacterial removal from dentinal tubules after cleaning and shaping of the root canal system. ${ }^{10}$

Irrigation systems which increase the penetration depth of irrigants in dentinal tubules and the root canal while encountering minimal apical extrusion of irrigants, eliminating cytotoxic effects on periapical tissues, exhibit superior effects. ${ }^{11,12}$

Dental lasers have probable photo biologic effects including photoacoustic, photochemical and photothermal effects. ${ }^{13}$

Laser activated irrigation (LAI) was introduced with the aim of increasing irrigant activation. This technique causes acoustic cavitation, defined by the formation of large oval vapor bubbles which will expand and implode subsequent to the use of ablative lasers in an aqueous environment. It has been shown that these vapor bubbles may increase the volume up to 1600 times. Expansion creates high pressure and the pressure causes the expulsion of fluid. When the bubbles implode after 100-200 ms, the pressure declines. By means of subsequent re-entry of fluid into the root canal, the secondary effects of cavitation are created. Thus, the laser acts as a fluid pump. ${ }^{14}$

In the steps of cleaning and shaping the root canal system, application of laser has some advantages such as debris re- moval, smear layer removal and bactericidal effects. ${ }^{15}$ Few studies have reported adverse effects from this type of laser. It also has been confirmed that this laser can strengthen disinfection and sterilization due to thermal effects. ${ }^{16}$ This laser can reduce the post-operative pain owing to the effective removal of bacteria in the apical region of the root canal. ${ }^{17}$ Other benefits include melting of dentin and sealing the dentinal tubules, ${ }^{18}$ removal of debris and smear layer from root canal walls and tissue vaporization. ${ }^{19}$

Little information exists about the effects of Neodymium-Doped Yttrium Aluminum Garnet (Nd:YAG) LAI on the penetration depth of sodium hypochlorite. And no study has compared this laser with other methods. The aim of this Ex vivo study was to evaluate the extent of penetration of sodium hypochlorite into dentinal tubules by passive ultrasonic agitation (PUA), Nd:YAG LAI and compare them with conventional clinical methods and determine the more effective and practical irrigation method for endodontic treatments.

\section{Methods}

Seventy-two extracted human anterior mandibular teeth with one root canal were selected. The roots of teeth were mature and straight, without caries, resorption and observable cracks under $3 \mathrm{X}$ magnification. The teeth were extracted due to periodontal problems. The teeth were stored in normal saline and disinfected by chloramines T with 1:7 proportion. The crowns of teeth were cut off by diamond discs with a thickness of $1 \mathrm{~mm}$ (D \& Z, Cologne, Germany) to provide samples with $19 \mathrm{~mm}$ length from the apical foramen. The position of apical foramen was determined by a stainless steel K-type hand file \#15 (Dentsply, Maillefer, Ballaigues, Switzerland). After seeing the tip of file in the apical foramen, $1 \mathrm{~mm}$ was reduced from the length of file and that file length was considered as working length.

Then, the samples were randomly divided into 4 groups: Group 1: Conventional irrigation (CI): First, the root canals were irrigated with $2 \mathrm{ml} \mathrm{5.25 \%} \mathrm{NaOCl}$ by an endodontic irrigating syringe and 30 gauge needle (Max-iProbe; Dentsply, Rinn, Elgin, IL) for 20 seconds. The tip of the needle was placed $1 \mathrm{~mm}$ to the apical foramen and was moved up and down in the apical third with latitude of $4 \mathrm{~mm}$. Then $\mathrm{NaOCl}$ was left in the root canal for 20 seconds without any movement, and the canals were irrigated again for 20 seconds as described previously with 30 gauge Max-i-Probe needle. Finalirrigation was accomplished by normal saline for 60 seconds.

Group 2: CI plus smear layer removal: The canals were irrigated by $2 \mathrm{ml} 5.25 \% \mathrm{NaOCl}$ with a syringe and 30 gauge Max-i-Probe needle for 20 seconds as described previously in group 1 . Then for smear layer removal, $2 \mathrm{ml}$ of $17 \%$ ethylenediaminetetraacetic acid (EDTA) (Apadana Tak Co, Tehran, Iran) was used for one minute followed by 2 $\mathrm{ml} 5.25 \% \mathrm{NaOCl}$ for another minute. Final irrigation was accomplished by normal saline for 60 seconds. This group was considered as the gold standard group.

Group 3: PUA group: The root canals were irrigated by 
$2 \mathrm{ml} 5.25 \% \mathrm{NaOCl}$ with a syringe and 30 gauge Max-iProbe needle for 20 seconds. Then, a stainless steel NSK $\mathrm{U}$ file $33 \mathrm{~mm} \# 20$ mounted at E11 ultrasonic tip (Varios, NSK Nakanishi Inc., Kanuma, Japan) was activated for 20 seconds with an ultrasonic device (Varios 350, NSK Nakanishi Inc., Kanuma, Japan) with the power set on E4 to agitate the solution. The tip of file was placed $1 \mathrm{~mm}$ above apical foramen according to the method explained by De Moor et al. ${ }^{20}$ Then, the canals were irrigated by $2 \mathrm{ml} 5.25 \%$ $\mathrm{NaOCl}$ with a syringe for 20 seconds. Final irrigation was accomplished by normal saline for 60 seconds.

Group 4: LAI group: The teeth were filled by $2 \mathrm{ml} \mathrm{5.25 \%}$ $\mathrm{NaOCl}$ with syringe and 30 gauge Max-i-Probe needle for 20 seconds. Then, the Nd:YAG laser (Fidelis plus, Fotona; Ljubljana, Slovenia) with $300 \mu \mathrm{m}$ endodontic fiber and $100 \mathrm{~mJ}$ energy pulse and power of $2 \mathrm{~W}$, frequency of $20 \mathrm{~Hz}$ and pulse width of $100 \mu \mathrm{s}$ (VSP: Very Short Pulse) was activated for 20 seconds. The fiber was placed in the apical part of the root canal for 5 seconds and moved in spiral motion out of the root canal. Then the fiber was reinserted in the root canal and this procedure was repeated for 4 more times (total time $=20$ seconds). The laser was only fired during fiber removal from the root canal according to Blanken et al..$^{21}$ Then, the root canal was irrigated with $2 \mathrm{ml} 5.25 \% \mathrm{NaOCl}$ for 20 seconds with syringe and 30 gauge Max-i-Probe needle. Final irrigation was accomplished by normal saline for 60 seconds.

After irrigation, the samples were separated in half. One half was randomly selected and then the surface layer was grounded with a thickness of approximately 100 microns by a soft abrasive paper and grinding machine (Malek Teb, Tehran, Iran). Then the created surfaces were evaluated with stereomicroscope Model LA-SZM45-B1 (Nanjing Sunny Optical Instrument Co., Ltd, Nanjing, China) with 20X magnification. Images were saved in JPEG format by Dinocapture 2.0 software using AM423X Dino-Eye digital eyepiece and RL-L64 LED light source LED model. The depth of the bleached zone (depth of penetration of sodium hypochlorite) was calculated in microns.

\section{The Method of Calculating the Bleached Zone}

The captured images from stereomicroscopic with 20X magnifications, were opened with Photoshop software. The limits of bleached zone were determined by polygonal lasso tool, number of selected pixels was read in Photoshop histogram menu and likewise, height of canal interior wall was read based on pixel in Info menu. The penetration depth of irrigation solution was calculated according to the following formula based on pixel:

$b=\frac{\text { Total pixels }}{a}$

( $b=$ Average penetration depth of irrigation solution, $a=$ Height of canal interior wall) (Figure 1).

For images with 72 dpi resolution, every 100 pixels were equivalent to $35.28 \mathrm{~mm}$ and by dividing that to the magnification, the real size which is convertible to micron was obtained and in that way, the average, minimum and max-
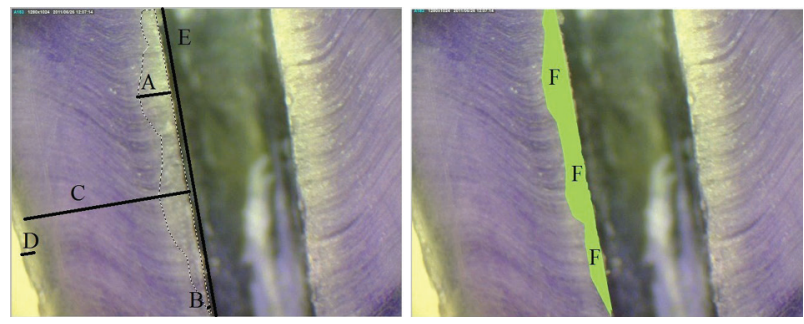

Figure 4. Determining Bleached Level by Photoshop Software. A) Maximum penetration depth of sodium hypochlorite, B) Minimum penetration depth of sodium hypochlorite, C) Dentin, D) Cementum, E) Height of canal interior wall, and F) Limits of bleached zone.

imum penetration in the total penetrated surface was obtained. The results of penetration depth were announced by two trained blind observers in such a way that they came to the same conclusion after some disagreement. Afterwards, statistical analysis was performed.

For data analysis, quantitative variables were described as average and standard deviation and qualitative variables as raw abundance and relative abundance.

To compare the average penetration depth of sodium hypochlorite among four study groups, analysis of variance was used and for pair wise comparison, Dunnett T3 test was applied due to non-homogenous variance of data. A statistical significant limit of 0.05 was considered for this purpose.

\section{Results}

Average penetration depth of sodium hypochlorite in the coronal third was different among the four groups $(P<0.001)$. The average penetration depth of sodium hypochlorite was significantly different when comparing the CI+smear layer removal group (309.81 microns) with CI groups (249.93 microns ) and PUA (260.56 microns) groups $(P<0.001$ and $P=0.001$ respectively). Also, the average penetration depth of sodium hypochlorite was significantly different when comparing the LAI group (293.03 microns) with CI (249.93 microns) and PUA (260.56 microns) groups $(P<0.001$ and $P=0.004$ respectively).

Average penetration depth of sodium hypochlorite in the middle third was different among the four groups under study $(P<0.001)$. A statistically significant difference was seen when comparing the average penetration depth of sodium hypochlorite between the CI+smear layer removal group (212.7 microns) with CI groups (163.9 microns) and PUA (188.12 microns) $(P<0.001$ and $P=0.04$ respectively). A statistically significant difference was also seen when comparing the average penetration depth of sodium hypochlorite between the CI groups (163.9 microns) with LAI groups (207.79 microns) and PUA (188.12 microns) $(P<0.009$ and $P=0.001$ respectively).

The average penetration depth of sodium hypochlorite in apical one third was also different in the four experimental groups $(P<0.001)$. A statistically significant difference was seen when comparing the average penetration depth 
of sodium hypochlorite between CI+smear layer removal group (175.41 microns) with CI groups (42.07 microns), PUA (71.61 microns) and LAI (90.2 microns) $(P<0.001)$. The average penetration depth of sodium hypochlorite in group CI (42.07 microns) had a significant difference with that of the LAI (90.2 microns) and PUA (71.61 microns) groups $(P<0.001)$. Furthermore, a significant difference was seen in the average penetration depth of sodium hypochlorite between LAI (90.2 microns) and PUA (71.61 microns) groups $(P=0.05)$. The results are shown in Table 1.

\section{Discussion}

Studying the extent and measuring the penetration depth of sodium hypochlorite in dentin is not possible in vivo due to practical and moral limitations. Therefore, to standardize the study, human extracted teeth were used (ex vivo).

William et $\mathrm{al}^{22}$ used the motile microorganisms model to study penetration depth of smear layer in an in vitro study. They reported that in severely infected root canals, bacteria might be found in depth of dentinal tubules and the smear layer covering the root canal walls following endodontic instrumentation contained bacteria and remains of necrotic pulp tissues.

Also, the presence of smear layer can prevent antiseptics and root canal fillers from entering dentinal tubules. ${ }^{23}$

Nair et $\mathrm{al}^{24}$ showed that $88 \%$ of root canals of mesial roots of one-visit treated mandibular molars revealed residual infection after instrumentation, irrigation with sodium hypochlorite, and obturation. Due to these limitations, searching for the enhancement of the results of sodium hypochlorite irrigation by devices or a better root canal irrigant must be continued. ${ }^{24}$

Likewise, a study by Hata et al showed that instrumentation and irrigation with 5\% sodium hypochlorite followed by $15 \%$ EDTA using a syringe was the most effective irrigation technique for smear layer removal. ${ }^{25}$ Therefore, in the current study, conventional irrigation and smear layer removal by the aforementioned protocol was considered as the gold standard for comparison.

Since mechanical instrumentation only eliminates $50 \%$ of bacteria from root canal, irrigating solutions are required to eliminate microbiota from the zones out of reach of instruments. ${ }^{26}$ Different activation techniques are recommended for promoting the efficiency of irrigating solutions. Some of them include agitation by hand file, Gutta Percha, mechanical agitation by plastic instruments, also sonic and ultrasonic agitation. ${ }^{27}$

Minamisako et al have shown the efficiency of Nd:YAG laser in eliminating debris, smear layer and pulp tissues. ${ }^{19}$ Therefore, in the current study, Nd:YAG was used with 2 W power, $20 \mathrm{~Hz}$ frequency and 100 microsecond pulse width (VSP: very short pulse) for 20 seconds. Longer irradiation time with less output power decreases the risk of tissue damage due to significantly less temperature increase. On the other hand, to diffuse the heat generated from laser radiation and in tensify the effects of laser radiation, canals may be filled with sodium hypochlorite or EDTA. ${ }^{19}$

Mello et $\mathrm{al}^{28}$ concluded that constant irrigation with $5 \mathrm{ml}$ of EDTA for 3 minutes can effectively remove smear layer. In vivo conditions such as moisture, temperature and dentinal tubule content may impress the penetration depth to dentinal tubules. ${ }^{29}$ Likewise, the penetration depth of irrigating solutions into dentinal tubules, and as a result, their bactericidal effects are clearly impressed by the presence or lack of smear layer. It has been indicated that smear layer formation, decreases penetration into root dentin about $25 \%-49 \% .^{30}$

In some studies, dye penetration in sodium hypochlorite solution has been used as an index for evaluating penetration, but, there is doubt whether there is a conformity between the penetration depth of sodium hypochlorite and dye penetration depth or not. It has been reported that the surface tension of sodium hypochlorite limits its capabil-

Table 1. Statistical Indicators for the Penetration Depth of Sodium Hypochlorite in the Coronal, Middle and Apical Third of Four Experimental Groups (Based on Microns)

\begin{tabular}{|c|c|c|c|c|}
\hline Groups & LAI & PUA & $\mathrm{CI}+$ Smear Layer Removal & $\mathrm{Cl}$ \\
\hline \multicolumn{5}{|l|}{ Coronal } \\
\hline Mean & $293.03^{a, b}$ & $260.56^{a, c}$ & $309.81^{c, d}$ & $249.93 \mathrm{~b}, \mathrm{~d}$ \\
\hline SD & 17.56 & 31.15 & 39.01 & 24.57 \\
\hline Min & 245.46 & 202.14 & 215.96 & 188.62 \\
\hline Max & 316.34 & 301.09 & 389.43 & 283.34 \\
\hline \multicolumn{5}{|l|}{ Middle } \\
\hline Mean & 207.79 e & $188.12^{\mathrm{f}, \mathrm{g}}$ & $212.7^{\mathrm{f}, \mathrm{h}}$ & $163.9^{e, g, h}$ \\
\hline SD & 33.49 & 23.22 & 27.28 & 18.17 \\
\hline Min & 140.65 & 140.25 & 175.76 & 129.76 \\
\hline Max & 269.12 & 245.45 & 279.13 & 190 \\
\hline \multicolumn{5}{|l|}{ Apical } \\
\hline Mean & $90.2^{i}$ & $71.61^{i}$ & $175.41^{i}$ & $42.07^{i}$ \\
\hline SD & 18.64 & 38.75 & 38.75 & 17.71 \\
\hline Min & 52.32 & 119.32 & 110.32 & 10.54 \\
\hline Max & 121.52 & 234.57 & 234.57 & 70.09 \\
\hline
\end{tabular}

Abbreviations: LAI, laser activated irrigation; PUA, passive ultrasonic agitation; $\mathrm{Cl}$, conventional irrigation

Similar alphabetical superscripts indicate significant difference amongst the groups. 
ity to distribute inside the root canal. ${ }^{31}$ Adding some acid fuchsin to sodium hypochlorite may have some effect on its surface tension which should come into consideration in future studies. Due to the small molecular size of sodium hypochlorite (molecular weight of $\mathrm{NaOCl}$ solved in distilled water is $74.45 \mathrm{~g} /$ mole), its penetration depth may be higher than that of the dye itself (acid fuchsin molecular weight is $585.54 \mathrm{~g} / \mathrm{mole}$ ). Therefore, advancement of the solution in the root canal is assessed indirectly and the results may not bedefinite. ${ }^{32}$

Previous studies indicate that there are some certain dyes that may easily penetrate into the whole depth of dentin even without removing root surface cementum. ${ }^{33}$ Crystal violet and safranin red showed the same results in dye and sodium hypochlorite penetration studies. ${ }^{34}$

The reason for selecting crystal violet in the current study was better observation under stereomicroscope. Because sodium hypochlorite is a strong oxidant, it whitens the purple color of crystal violet and reveals the clear natural color of dentin. The width of the discolored zone was easily recorded and considered as the penetration depth of sodium hypochlorite. After exposing floated samples in crystal violet to sodium hypochlorite, 100 micron was removed from surface of dentin samples by abrasive paper to let the dentin zones that were only under the impression of sodium hypochlorite via dentinal tubules come up. Of course, it is assumed that coloring the walls of dentinal tubules by dye may change liquid penetration patterns by changing dentine surface features. ${ }^{34}$

Penetration depth and presence of bacteria in various surfaces of dentin and inside dentinal tubules have been studied by some researchers. For instance, Peters et al in an in vivo study showed that in $62 \%$ of cases, bacteria grew up to the cemental surface of the root and in $24 \%$ of cases, over $50000 \mathrm{CFU} / \mathrm{g}$ were seen on root cementum surface. ${ }^{35}$ Further studies, reported the maximum penetration depth of bacteria in the pulpal dentin wall to be up to $0.25 \mathrm{~mm} .{ }^{36}$ On the other hand, Siqueira et al observed bacterial cells up to an approximate depth of 300 microns and believed that low penetration depth was more conventional. ${ }^{13}$

Also, resistant infections resulting from reproduction of the remaining bacteria inside dentinal tubules are one of the probable reasons for treatment failure. ${ }^{37}$

Based on the aforementioned studies, increasing the penetration depth of irrigation solutions inside dentinal tubules may be effective in promoting the success rate of endodontic treatments and decreasing failure in the treatment of resistant infections. According to the findings of the present study, the average penetration depth of sodium hypochlorite in the coronal third was different among the four studied groups. The highest penetration depth was seen in the CI+smear layer removal group (309.81 microns) and the lowest was seen in the CI group (249.93 micron). Differences were statistically significant $(P<0.001)$. The differences seen in the coronal third do not seem to be of clinical significance.

In the middle third, the highest penetration depth was seen in the CI + smear layer removal group (212.70 micron) and the lowest was seen in the CI group (163.90 micron). The difference was statistically significant $(P<0.001)$.

In terms of clinical significance, it seems that by approaching the apical zone, such differences(about 60 microns) clearly become more important and effective. Similar to the middle third zone, the average penetration depth of sodium hypochlorite in LAI group was 207.79 microns and that amount did not show a significant difference with the gold standard group (212.70 microns). On the contrary, the difference seen between the PUA and gold standard group was significant. It can be concluded that in middle and coronal third, only LAI was as effective as the gold standard protocol in increasing the penetration depth of irrigation solution.

In apical third, similar to that of the middle and coronal third, the highest penetration depth belonged to the CI + smear layer removal group, and the CI group showed the lowest penetration depth (175.41 and 40.07 microns respectively). In the apical third, the average penetration depth of sodium hypochlorite in the PUA and LAI groups were 71.61 and 90.20 micron respectively, which had a significant difference with the gold standard group (175.41 micron). An 85-105 microns difference has a clear clinical impact in the apical third zone.

What comes into spotlight in all the aforementioned comparisons is that EDTA was never used in CI, PUL and LAI techniques and probably its significant role might be the reason for such significant difference. In this study, despite of implementing activation techniques, $\mathrm{NaOCl}$ did not show complete penetration in any of the samples and this can justify failure in ideal root canal treatments.

In this study, the penetration depth of sodium hypochlorite was reported to be in a range of 40 to 309 microns. Ando and Hoshino showed presence of bacteria in dentinal tubules up to the half way between root canal and cementodentinal junction (CDJ) ${ }^{38}$ Haapasalo et al reported that Enterococcus faecalis quickly attacked tubules and in some blocks, they could penetrate up to a depth of 1000 microns after three weeks incubation. ${ }^{37}$ Therefore, access to all bacteria in all cases by irrigation with sodium hypochlorite would be difficult or almost impossible and various factors such as viscosity, time, temperature, surface tension, needle gauge and needle head depth position may affect the penetration depth of irrigation solutions such as $\mathrm{NaOCl}{ }^{34}$

In the study of Zou et $\mathrm{al},{ }^{34}$ the highest amount of penetration depth of $6 \%$ sodium hypochlorite was 300 microns which was seen after application of this solution for 20 minutes in $45^{\circ} \mathrm{C}$. It was shown that temperature, time and viscosity were variables affecting the penetration depth of sodium hypochlorite into dentinal tubules. The deepest penetration took place when all the three variables existed simultaneously contributing to intensification. ${ }^{34}$

De Gregorio et $\mathrm{l}^{39}$ evaluated the penetration of irrigation solution into synthetic accessory canals. Dissimilar to the results of the current study, they concluded that adding 
EDTA did not lead to better penetration of irrigation solution into synthetic accessory canals. The use of accessory canals instead of dentinal tubules may be the reason for such differences.

Minamisako et al reported that Nd:YAG laser was capable of removing smear layer, debris and pulpal tissues even without black initiator ink. ${ }^{19}$ The results of present study indicated that the penetration depth of sodium hypochlorite in the coronal and middle sections did not show significant differences when comparing the use of laser with the gold standard; whereas, in apical third, the LAI group, showed a significant difference when compared to the gold standard group in such a way that the average penetration depth of sodium hypochlorite was 90.2 microns in the $\mathrm{v}$ group and 175.41 micron in the gold standard group. The lower penetration depth can be attributed to higher amounts of the remaining smear layer, the melting effect of Nd:YAG laser on dentin and occlusion of dentinal tubule orifices.

According to the results of the current study it can be concluded that the standard protocol for smear layer removal leads to a more effective elimination of smear layer and deeper penetration of irrigation solution. It should be noted that penetration of irrigating solution into dentinal tubules does not necessarily indicate its sufficient concentration for eliminating microorganisms, ${ }^{40}$ which is of more importance in infected root canals.

Lloyd et $\mathrm{al}^{41}$ showed that laser-activated photon-induced photo-acoustic streaming irrigation can eliminate debris from complex canal spaces of mandibular molars at a significantly greater level in comparison with standard needle irrigation.

Further studies regarding effective elimination of biofilm and microorganism inside dentinal tubules using other types of lasers with various settings and environments are highly recommended.

\section{Conclusion}

The use of PUA and LA resulted in less smear layer elimination and consequently less penetration of irrigating solutions. CI + smear layer removal can be considered as the gold standard technique for deeper penetration of $\mathrm{NaOCl}$ in dentinal tubules and techniques that do not remove smear layer cannot be considered as a good alternate for it.

\section{Ethical Considerations}

The teeth used in this study were extracted due to periodontal problems.

\section{Conflict of Interest}

The authors has no conflict of interest to declare.

\section{References}

1. Clegg MS, Vertucci FJ, Walker C, Zand V. The effect of exposure to irrigant solutions on apical dentin biofilms in vitro. J Endod. 2006;32(5):434-437. doi:10.1016/j. joen.2005.07.002.
2. Chow TW. Mechanical effectiveness of root canal irrigation. J Endod. 1983;9(11):475-479. doi:10.1016/ s0099-2399(83)80162-9.

3. Baker NA, Eleazer PD, Averbach RE. Scanning electron microscopic study of the efficacy of various irrigation solutions. J Endod. 1975;1(4):127-135.

4. Cheung GS, Stock CJR. In vitro cleaning ability of root canal irrigants with and without Endosonics. Int Endod J. 1993;26(6):334-343. doi: 10.1111/j.1365-2591.1993. tb00766.x

5. Foster KH, Kulild JC, Weller RN. Effect of smear layer removal on the diffusion of calcium hydroxide through radicular dentin. J Endod. 1993;19(9):136-140.

6. Nielsen BA, Baumgartner JC. Comparision of the Endovac system to needle irrigation of the root canals. J Endod. 2007;33(5):611-615. doi:10.1016/j.joen.2007.01.020.

7. Chopra S, Murray PE, Namerow KN. A scanning electron microscopic evaluation of the effectiveness of the F-file versus ultrasonic activation of a K-file to remove smear layer. J Endod. 2008;34(12):1234-1235. doi:10.1016/j. joen.2008.07.006

8. Bahcall J, Oslen FK. Clinical introduction of a plastic rotary endodontic finishing file. Endo Prac. 2007;10(5):17-20.

9. Paragliola R, Franco V, Fabiani C, Mazzoni A, Nato F, Tay FR, et al. Final Rinse Optimization: Influence of Different Agitation protocols. J Endod. 2010;36(2):282-285. doi:10.1016/j.joen.2009.10.004

10. Wu Mk, Dummer PM, Wesselink PR. Consequences of and strategies to deal with residual post-treatment root canal infection. Int Endod J. 2006;39(5):343-356. doi:10.1111/ j.1365-2591.2006.01092.x.

11. Serper A, Ozbek M, Calt S. Accidental sodium hypochloriteinduced skin injury during endodontic treatment,. J Endod. 2004;30(3):180-181. doi:10.1097/00004770-20040300000013.

12. Bradford CE, Eleazer PD, Downs KE, Scheetz JP. Apical pressures developed by needles for canal irrigation. J Endod. 2002;28(9):333-335. doi:10.1097/00004770200204000-00019

13. Siqueira JF, Rocas IN, Lopez HP. Patterns of microbial clonization in primary root canal infections. Oral Surg Oral Med Oral Pathol Oral Radiol Endod. 2002;93(12):174-178.

14. Van der Sluis LM, Wu MK, Versluis M, Wesselink PR. Passive ultrasonic irrigation of the root canal: a review of the literature. Int Endod J. 2007;40(6):415-426.

15. Stabholz A, Sahar-Helft S, Moshonov J. lasers in endodontics. Dent clin north Am. 2004;48(4):809-832. doi:10.1016/j.cden.2004.05.012.

16. Wang Q, Zhang C, Yin X. Evaluation of bactericidal effect of Er;Cr:YSGG and Nd:YAG lasers in experimentally infected root canals. J Endod. 2007;33(7):830-832. doi:10.1016/j. joen.2007.03.017.

17. Koba K, Kimura Y, Matsumoto K, Takeuchi T, Ikarugi T, Shimizu T. A histopathologial study of the morphological changes at the apical seat in the periapical region after irradiation with a pulsed Nd:YAG laser. Int Endod J. 1998;31(6):415-420. doi:10.1046/j.13652591.1998.00177.x.

18. Miserendino LJ, Levy GC, Rizoiu IM. Effects of the Nd:YAG laser on the permeability of root canal wall dentin. J Endod. $1995 ; 21(2): 83-87$

19. Minamisako MC, Kinoshita JL, Matsumoto K, Stolf DP, Marques JL. A study on root canal cleaning by Nd:YAG 
laser with black dye solution. J Oral Laser Applications. 2009;9(2):101-109.

20. De Moor RJ, Torbeyns D, Meire M. Lasers in endodontics. Part 2: root canal wall cleanliness and modification. Endod Pract Today. 2009;3:19-33.

21. Blanken J, De Moor RJ, Meire M, Verdaasdonk R. Laser induced explosive vapor and cavitation resulting in effective irrigation of the root canal. Part 1: a visualization study. Lasers Surg Med. 2009;41(7):514-519. doi:10.1002/ lsm.20798.

22. William S, Goldman M. Penetrrability of the smeared layer by a strain of Proteus vulgaris. J Endod. 1985;11(5):385388. doi:10.1016/s0099-2399(85)80026-1.

23. Goldberg F, Abramovich A. Analysis of the effect of EDTAC on the dentinal walls of the root canal. J Endod. 1977;3(5):101-105. doi:10.1016/s0099-2399(77)80203-3.

24. Nair BG, Reddy KA. Advances in root canal disinfection. J Pharm Biomed Sci. 2011;5(24):1-3.

25. Hata G, Hayami S, Weine FS, Toda T. Effectiveness of oxidative potential water as a root canal irrigant. Int Endod J. 2001;34(12):308-317. doi:10.1046/j.13652591.2001.00395.x

26. Ciucchi B, Khettabi M, Holz J. The effectiveness of different endodontic irrigation procedures on the removal of smear layer: A scanning electron microscopic study. Int Endod J. 1989;22(9):21-28. doi:10.1111/j.1365-2591.1989.tb00501.x.

27. Gu LS, Kim JR, Ling j, Choi KK, Pashley DH, Tay FR. Review of contemporary irrigant agitation techniques and devices. J Endod. 2009;35(6):791-804. doi:10.1016/j. joen.2009.03.010.

28. Mello I, Kammerer BA, Yoshimoto D, Macedo MC, Antoniazzi $\mathrm{JH}$. Influence of final rinse technique on ability of Ethylenediaminetetraacetic acid of removing smear layer. J Endod. 2010;36(3):512-514. doi:10.1016/j. joen.2009.11.004.

29. Kokkas AB, BoutsioukisACh, Vassiliadis LP, Stavrianos $\mathrm{CK}$. The influence of the smear layer on dentinal tubule penetration depth by three different root canal sealers : an in vitro study. J Endod. 2004;30(2):100-102. doi:10.1097/00004770-200402000-00009.

30. Fogel HM, Pashley DH. Dentin permeability : effects of endodontic procedures on root slabs. J Endod. 1990;16(9):442-445. doi:10.1016/s0099-2399(06)81888-1.
31. Cunningham WT, Cole JS 3rd, Balekjian AY. Effect of alcohol on the spreading ability sodium hypochlorite endodontic irrigant. Oral surgery, Oral medicine and oral Pathology. 1982;54(12):333-335.

32. Hauser V, Braun A, Frentzen M. Penetration depth of a dye marker into dentin using a novel hydrodynamic system (RinsEndo). Int Endod J. 2007;40(8):644-652. doi:10.1111/ j.1365-2591.2007.01264.x

33. Paque F, Luder HU, Sener B, Zehnder M. Tubular sclerosis rather than smear layer impedes dye penetration into the dentine of endodontically instrumented root canals. Int Endod J. 2006;39(1):18-25.

34. Zou L, Shen Y, Li W, Haapasalo M. Penetration of Sodium Hypochlorite into dentin. J Endod. 2010;36(5):793-796.

35. Peters LB, Wesselink PR, Buijs JF, Winkelholf AJ. Viable bacteria in root dentinal tubules of teeth with apical periodontitis. J Endod. 2001;27(5):76-81. doi:10.1097/00004770-200102000-00002.

36. Schafer E, Bossmann K. Antimicrobial efficacy of chlorhexidine and two calcium hydroxide formulations against Entrococcus fecalis. J Endod. 2005;31(9):53-56. doi:10.1097/01.don.0000134209.28874.1c.

37. Haapasalo M, Qrstavik D. In vitro infection and disinfection of dentinal tubules. J Dent Res. 1987;66(5):1375-1379. doi: 10.1177/00220345870660081801.

38. Ando N, Hoshino E. Predominant obligate anaerobes invading the deep layers of root canal dentin. Int Endod J. 1990;23(12):20-27. doi:10.1111/j.1365-2591.1990. tb00798.x.

39. De Gregorio C, Steves R, Cisneros R, Heilborn C, Cohenca N. Effect of EDTA, sonic and ultrasonic activation on the penetration of sodium hypochlorite into simulated lateral canals: An in vitro study. J Endod. 2009;35(6):891-895. doi:10.1016/j.joen.2009.03.015.

40. Buck R, Eleazer PD, Staat RH. In vitro disinfection of dentinal tubules by various endodontic irrigants. J Endod. 1999;25(9):786-788. doi:10.1016/s0099-2399(99)80297-0.

41. Lloyd A, Uhles JP, Clement DJ, Garcia-Godoy F. Elimination of intracanal tissue and debris through a novel laser-activated system assessed using high-resolution micro-computed tomography: a pilot study. J Endod. 2014;40(4):584-587. doi:10.1016/j.joen.2013.10.040 